V. Aus der medicinischen Universitätspoliklinik des Herrn Prof. Dr. Jürgensen in Tübingen.

\title{
Ueber septische Allgemeinerkrankung nach chronischer Endocarditis.
}

Von Dr. Rudolf Paulus aus Stuttgart.

(Schluss aus No. 17).

Betrachten wir nun zunächst die Einzelsymptome dieses Falls:

Dass ein schwereres Allgemeinleiden vorliegt, zeigt schon der von Anfang an vorhandene Rückgang der Ernährung. Es darf dieselbe wohl weder auf die immerhin nicht unbedeutenden dyspeptischen Störungen, noch auf das anhaltende Fieber zurückgeführt werden, beide haben sicherlich einen Einfluss geübt, aber aus ihnen allein lässt sich die immer mehr zunehmende Abmagerung kaum ableiten. Alle Beobachtungen über septische Infection lehren, dass diese mit erheblicher Schädigung des Gesammtorganismus einhergeht, und so wird es auch hier gewesen sein. Ein wie grosser Einfluss den einzelnen Schädigern zuzumessen ist, möge dahingestellt sein

Während der ganzen Dauer der Beobachtung war Erhöhung der Körperwärme vorhanden. Die Ergebnisse der 86 Tage lang regelmässig vorgenommenen Messungen sind in der nachstehenden Tabelle zusammengestellt.

\begin{tabular}{|c|c|c|c|c|c|c|}
\hline Zeit der Beobachtung & Maximum & Minimum & Mittel & \multicolumn{3}{|c|}{ Tageszeit } \\
\hline 6. bis 19. August & 38,8 & 37,5 & 37,95 & morgens & $8 \mathrm{U}$ & \\
\hline & & & & abends & 6 & \\
\hline \multirow[t]{2}{*}{ 20. August bis 2. September } & & 37,4 & 37,85 & morgens & 8 & \\
\hline & & $\left.36,9^{1}\right)$ & & abends & $\mathbf{6}$ & \\
\hline \multirow[t]{2}{*}{ 3. bis 16. September } & & 37 & 37 & morgens & 8 & \\
\hline & & & & abends & 6 & \\
\hline \multirow[t]{2}{*}{ 17. bis 30 . September } & & & 37 , & morgens & 8 & \\
\hline & & & & abends & 6 & \\
\hline \multirow[t]{2}{*}{ 1. bis 14. October } & & 37,5 & 37 & morgens & 8 & \\
\hline & & 38 , & 38 , & abends & 6 & \\
\hline \multirow[t]{2}{*}{ 15. bis 28 . October } & & 37,6 & 38,11 & morgens & 8 & \\
\hline & & & 38 & abends & 6 & \\
\hline \multirow[t]{3}{*}{ 29. Octbr. bis 11. Novbr. } & & & 38 & morgens & 8 & \\
\hline & & & & mittags & 2 & \\
\hline & & & & abends & 6 & \\
\hline \multirow[t]{3}{*}{ 12. bis 25 . November } & 9,0 & 37,1 & & morgens & 8 & \\
\hline & & & & mittags 1 & 2 & \\
\hline & & & 39,92 & abends & 6 & \\
\hline \multirow[t]{3}{*}{ 26. Novbr. bis 9. Decbr. } & 38 & 37,3 & 37,79 & morgens & 8 & \\
\hline & & & & mittags 1 & 2 & \\
\hline & & 38 & 38 & abends & 6 & \\
\hline \multirow[t]{3}{*}{ 10. bis 23. December } & 38 & 37,3 & 37,64 & morgens & 8 & \\
\hline & & 37 & 38 , & mittags & 2 & \\
\hline & & 38 & 39,13 & abends & 6 & \\
\hline \multirow[t]{2}{*}{ 24. December bis 6. Januar } & 38,4 & 37 & 37,77 & morgens & 8 & \\
\hline & & 37 & 38,25 & mittags & 2 & \\
\hline \multirow{3}{*}{ 7. bis 15. Januar ${ }^{3}$ ) } & & $\begin{array}{l}38,9 \\
37,2\end{array}$ & & abends & $\begin{array}{l}6 \\
8\end{array}$ & \\
\hline & & & 38,27 & mittags & 2 & \\
\hline & 39,5 & 37,8 & 38,74 & abends & 6 & \\
\hline
\end{tabular}

Kurz wäre das Fieber als ein anhaltendes, mässig hohes, nicht stark remittirendes zu bezeichnen.

Das Mittel aus sämmtlichen Morgenmessungen rom 6. August bis zum 6. Januar beträgt $37,9^{\circ}$

Das Mittel aus sämmtlichen $\mathrm{Abendmessungen} \mathrm{während} \mathrm{dieser}$ Zeit 38,70 .

Das ohne Arzneiwirkung beobachtete absolute Maximum war $39,7^{0}$, das absolute Minimum 37, $1^{0}$.

Die vom 29. October bis zum 6. Januar regelmässig bestimmten

1) Nach $1 \mathrm{~g}$ Phenacetin; unbeeinflusst: 37,7

2) Nach $3 \mathrm{~g}$ Natr. salicylic.; unbeeinflusst: 38,2 .

3) Da sämmtliche Diakonissen an Influenza erkrankt waren, wurde während der letzten zwei Wochen nicht mehr gemessen. 
Mittagtemperaturen lieferten das Mittel von $38,4^{0}$, die Einzeltage zeigen, wie wir das bei dieser Erkrankung sehr häufig, fast regelmässig finden, bald eine höhere Temperatur am Morgen oder Mittag als am Abend, bald eine der Norm entsprechende Vertheilung über die Tageszeiten.

Alles in allem ist in dem vorliegenden Falle die Abweichung von der Norm nicht so gross, wie es meist beobachtet wird. $\mathrm{Be}-$ merkenswerth und hervorzuheben ist, dass die in der Krankengeschichte erwähnten frischen Localisationen in den Knochen, in den Gelenken und an anderen Orten nicht mit höheren Temperatursteigerungen einhergehen, ja nicht einmal sich in der Curve verrathen. Es muss dies als durchaus ungewöhnlich bezeichnet werden. Ebenso, dass einmal eine Gabe von $3 \mathrm{~g}$ Natr. salicylic. und ein anderes mal eine solche von $1 \mathrm{~g}$ Phenacetin die Körperwärme nicht unerheblich herabzudrücken vermochten. Es darf nach unseren Erfahrungen als Regel betrachtet werden, dass Salicylsäure, Antipyrin, Antifebrin u. s. w. wohl die bei der Sepsis so hăufigen Knochen- und Gelenkschmerzen zu mildern vermögen, aber trotzdem auf die Temperatursteigerung, welche damit verbunden ist, ohne irgend welche Wirkung sind.

Das Herz zeigte sich von Anfang als der Sitz alter und der Herd neuer Erkrankung. Die früheren Aufzeichnungen erweisen, dass schon im Jahre 1878 eine Insufficienz der Mitralis und eine Stenose des linksseitigen Ostium venosum vorhanden gewesen war. Damals war die compensirende Hypertrophie des rechten Herzens festgestellt. Jetzt freilich war von einer solchen nicht viel vorhanden. Nur eine geringe Ausdehnung der Herzdämpfung nach rechts, keine Verstärkung des diastolischen Tons über der Pulmonalis. Allein geblieben waren die vorwiegend systolischen, indess auch bei der Diastole nicht fehlenden Geräusche an der Herzspitze. Dieselben waren fortwährend so laut und in ihrer Klangfarbe so wenig verschieden und wechselnd, dass an accidentelle Geräusche nicht wohl gedacht werden konnte. Die über der Aorta hin und wieder wahrnehmbaren Abweichungen von der Norm dagegen durften nicht ohne weiteres auf Erkrankung derselben bezogen werden, zumal da das Verhalten des Pulses dafür keinen Anhalt bot. Die am 13. Januar 1890 bemerkte Ausdehnung der Herzgrenzen nach rechts wuirde auf eine Erweiterung des rechten Ventrikels bezogen, welche durch den lang ausgezogenen Verlauf der Erkrankung ihre vollständige Erklärung fand: diastolische Dilatation der minder widerstandsfähigen Muskelmasse.

Dass am Herzen noch anderes als eine alte Veränderung am Eingang vom linken Vorhof in den linken Ventrikel vorhanden, ergaben theils mittelbare, theils unmittelbare Krankheitszeichen. Die stets vorhandene Unregelmässigkeit bei der Zusammenziehung, die angestrengte Thätigkeit des Herzens - sich durch die weithin vernehmbaren Muskeltöne verrathend und doch zn keiner voll ausreichenden Kreisung des Blutes (andauernde Cyanose, Kühlheit der Glieder, kleiner, leicht unterdrückbarer Puls) führend - weisen auf ungenügende Arbeitsleistung des Herzens hin, welche durch die alten Veränderungen allein nicht erklärbar waren. - Indirekt war für eine Erkrankung des Herzens, und zwar dafür, dass in ihm der Herd der bestehenden Sepsis zu suchen sei, geltend zumachen, dass die in ihm vorhandenen Störungen dauernde waren, ein anderes Organ aber nur voräbergehend ergriffen wurde. Die anhaltende Steigerung der Temperatur wies auf einen fortwährenden Fiebererreger hin - die klinische Erfahrung lehrt, dass ein solcher im Herzen selbst seinen Sitz zu haben vermag, ohne dass andere Störungen als die in unserem Falle vorhandenen an diesem sich zu zeigen brauchen. Von Zeit zu Zeit fand dann eine Verschleppung der infectiösen Mikroben aus dem Herzen statt, bei welcher andere Körpertheile in Mitleidenschaft gezogen wurden. Niemals aber haben dieselben eine beherrschende Stellung im Krankheitsbilde gewonnen.

Als Localisationen sind zu bezeichnen:

1. Erkrankungen der Gelenke und Knochen, welche schon bei der Aufnahme vorhanden waren, am 18. und 29. October, sowie am 23. Januar in stärkeren Anfällen sich zeigten, im Laufe des December wiederholt, aber viel schwächer auftraten. Hervorzuheben wäre noch die Betheiligung des rechten Ober- und Unterkiefers, sowie ihres Gelenkes, welche seltener ergriffen werden.

2. Auf der Haut traten am 18. und 19. October die von Jürgenseu beschriebenenen eigenartigen Erkrankungen auf. Wenige Tage vor dem Tode erscheineu dann noch Petechien.

3. Reibegeräusche über der Leber und der Milz sind wiederholt beobachtet worden, die Milz war dauernd vergrössert.

4. Eine eigenthümliche Erscheinung boten die Organe des Bauches dar. Grosse Reizbarkeit des Magens, welche sich durch übermässige Empfindlichkeit gegen die Einführung nicht vollkommen zusagender Nahrung zeigte, war neben einer recht hochgradigen
Empfindlichkeit gegen Druck schon von Anfang der Beobachtung zugegen und scheint länger bestanden zu haben. Gab doch die Kranke an, dass sie auswärts wegen eines Magengeschwüres behandelt worden sei. $O b$ ein solches wirklich vorhanden, wurde nach reiflicher Ueberlegung bei der Formulirung der klinischen Diagnose dahingestellt. - Neben diesen andauernden Beschwerden treten nun aber zweimal Erscheinungen von den Unterleibsorganen auf, die wenigsten das erstemal - am 8. November - eine geradezu bedrohliche Höhe erreichten. Erbrechen, heftige Schmerzen im Bauch, alle Zeichen des Collapsis, urplötzlich einsetzend und von tagelang andauernder Reizung des Peritoneums begleitet. Es war das Bild einer Visceralneuralgie, dem ähnlich, wie man es bei Gallenstein- oder höherem Grade von Darmkolik sieht. Aehnliches wiederholte sich am 3. Januar, aber nicht in diesem Maasse. Es ist schwer zu sagen, was hier vorgegangen ist. Aehnliches haben wir im Laufe septischer Infection zweimal gesehen, in dem einen Fall, der rasch tödtlich endete, wies die Section einen Darminfarct nach, in dem zweiten nicht mit dem Tode endenden, war eine erhebliche Darmblutung vorhanden. Leider wurde der Stuhl, den diese Kranke nach dem Anfall entleerte, aus Versehen weggeschüttet, ehe derselbe dem behandelnden Arzte zu Gesichte . kam.

Es ist nicht auszuschliessen, dass auch hier etwas der Art geschehen, dass möglicherweise ein gröberer Embolus in die rechte Niere eingedrungen ist, und das benachbarte Peritoneum auf irgend eine Weise in Mitleidenschaft gezogen wurde. Dafür könnte man die Thatsache geltend machen, dass an dem auf den Anfall folgenden Tage die rechte Nierengegend bei Druck schmerzhaft war, und dass bei der Section die rechte Niere "grösstentheils in gelbe, nekrotische Substanz umgewandelt und ibre Arterie durch grauröthliches Gewebe vollständig verschlossen war. ${ }^{*}$ Weiter, dass von diesem Tage an Eiweiss im Harn auftrat - hierauf ist indess schon darum weniger Gewicht zu legen, weil nicht von Tag zu Tag nach Eiweiss gesucht worden ist. Blut wurde im Harn nicht gefunden - allein bei vollständiger Verlegung ihrer Arterie hat diese ohnehin verkümmerte Niere kaum mehr secernirt. Dagegen ist zu bemerken, dass besonders die Ileocöcalgegend, und zwar schon am Tage des Anfalls schmerzhaft war, und dass sich hier für die Untersuchung am folgenden Tage vermehrtes Resistenzgefühl, vielleicht eine Koprostase zeigte. Es wurde hierauf zunächst der Nachdruck gelegt - nach der freiwilligen, 6 Tage später erfolgenden Kothentleerung nahmen in der That die Bescliwerden ab.

Während des zweiten Anfalls am 3. Januar war nun aber die linke Seite ergriffen, von Koprostase kounte keine Rede sein, und die Darreichung von Opium hatte die gleiche günstige Wirkung. Es soll nur daran erinnert werden, dass auch in der linken Niere Infarcte, wenn auch kleinere gefunden wurden. Auf die Deutung der beobachteten Erscheinungen möchten wir aber verzichten.

5. Die Erkrankung der rechten Lunge und ihrer Pleura blieb gering und trat erst während der letzten Wochen des Lebens auf. Die zu gleicher Zeit sich zeigenden Hirnsymptome sind wohl nicht auf die Infection unmittelbar zu beziehen, sondern hängen mit der schwächer werdenden Herzthätigkeit und dem Rückgang der Gesammternährung zusammen.

Fall 2. Carl Z. Kehrer, 58 Jahre alt, wurde am 2. März 1890 aufgenommen. Von früheren Krankheiten des Patienten ist nichts bekannt. Er erkrankte ganz plötzlich um Mitternacht vom 27. zum 28. Februar mit heftigem Schüttelfrost, während er Harn lassen wollte. Morgens steht er auf, aber er ist nicht imstande, sich auf den Füssen zu halten. Da er glaubte, es handle sich um die "Seuche" (Influenza), legt er sich zu Bett in der Hoffnung, die Sache würde sich von selbst wieder bessern. Erst am 2. März wurde um ärztlichen Beistand gebeten, weil der Kranke so sehr huste und nicht recht bei Bewusstsein sei. Um 12 Uhr Mittags am 2. März wird der sehr kräftige Patient unbesinnlich angetroffen. Er murmelt vor sich hin und nimmt von seiner Umgebung keine Notiz. Auf Anrufen giebt er Antwort, spricht einige verständige Worte, schweift aber bald wieder von der gegebenen Sache ab. Er will auf's Feld, da er noch viel $z u$ arbeiten habe etc.

Beim Entkleiden behufs Vornahme der Untersuchung versucht er sich selbst Hülfe zu geben, er sinkt aber nach wenigen kurzen Anstrengungen in's Bett zurück. Fäces und Harn lässt er unter sich gehen. Die Athmung geschieht angestrengt, ohne Pausen (39 Respirationen in der Minute). Puls 110, unregelmässig; es besteht starkes Atherom der peripheren Arterien.

Bei der flüchtigen Untersuchung scheint rechts hinten unten der Percussionsschall weniger voll als an der symmetrischen Stelle links. Bei der Auscultation war überall scharfes Vesiculärathmen mit einigen trockenen Rasselgeräuschen untermischt zu hören; nur an einer Stelle rechts hinten unten unter dem Angulus scapulae hörte man weiches Bronchialathmen und etwas feinblasiges in- und exspiratorisches Rasseln.

Die Herzdämpfung war eine der Norm entsprechende, die Herztöne schienen rein, sie konnten wegen des überaus lauten Athemgeräusches nur undeutlich wahrgenommen werden. Pupillenreaction vorhanden. Keine Schmerzen beim Betasten des Körpers. 
Abends 7 Uhr: Vollständig comatöser Zustand. Pupillen sehr eng, erweitern sich bei der Beschattung kaum. Auf ein kaltes Bad, das vorher gegeben wurde, soll Patient für kurze Zeit wieder lebhafter geworden seill. Beim Baden fiel es der Diakonissin auf, dass die Glieder des Patienten so steif und kaum zu beugen waren.

A bends 11 Ulır: Pupillen sehr weit. Sonst keine Veränderungen wahrnehmbar.

Am 3. März, morgens 3 Uhr, erfolgt der Tod, ohne dass Patient aus seinem Coma nochmals erwachte.

Temperaturtabelle:

$$
\begin{array}{cccl}
\text { 2. März, Nachmittags } 2 & \text { Uhr: } 40,8, \\
" & 4 & 41,3, \\
" & 6 & 41,3, \\
" & 8 & 71,0(\mathrm{Bad}) \\
\text { Nachts } 10 & 12 & 39,3, \\
& & 40,8,
\end{array}
$$

Section (Herr Prof. Baumgarten) 12 Std. p. m.: Grosse muskuIöse männliche Leiche von starkem Knoclienbau. Todtenstarre sehr stark entwickelt. Die Haut graugelb, nicht ikterisch, zeigt an der Brust und an verschiedenen Stellen des Unterleibs, sowie in den oberen Theilen der Schenkel, besonders nach der Glutaealgegend hin zahlreiche kleine Petechien. Die in der Augenlidspalte gelegenen Theile der Hornhaut sind stark eingetrocknet. Rechts eine Hernia cruralis. Die Leber in der Papillarlinie unter dem Rippenrand verborgen. Zwerchfellstand rechts am oberen Rand, links Mitte der dritten Rippe. Der Magen etwas stark ausgedehnt, tritt unter dem linken Leberlappen mehrere Finger breit hervor. Milz in normaler Lage.

Das Ilerz ziemlicli stark entwickelt. Die Muskulatur des rechten Ventrikels zeigt einen geringen Gradvon Hypertrophie. An der Mitralis findet sich eine geringe Insufficienz und Stenose, bedingt durch Schrumpfung des Klappenbindegewebes im Längs- und Querdurchmesser, sowie eine Verwachsung beider Segel durch eine verkalkte Narbenbrücke. Am freien unterell liand des Aortensegels der Mitralis einige kleine graue, höehstens stecknadelkopfgrosse fibrinähnliche Excrescenzen. Die Sehuenfåden des Aortensegels stark verdickt. $\Lambda \mathrm{n}$ der Grenzstelle $z$ wischen hinterer und linker Aortenklappe $z$ eigt sich eine schmierige, graue, wenig festhaftende Auflagerung von ganz geringer Ausdehnung. Das sonstige Gewebe der Seminularklappen der Aorta völlig normal. Der linke Ventrikel sonst nicht veründert. Blutungen oder embolische Herdchen im Myocard sind nicht zu entdecken.

Die reclite Lunge schlaff, durchweg lufthaltig, zeigt nur etwa in der Mitte des Unterlappens $z$ wei etwa 1 Quadratcentimeter grosseflache hämorrhagische Herde.

Die linke Lunge bietet ebenfalls ausser einer etwas grossen Hämorrlagie, die sich aunähernd in der Mitte des Unterlappens findet, keine pathologischen Verändernngen dar. Die Milz etwas vergrössert $(14 \mathrm{~cm}$ lang), von dunkelrother Färbung, etwas derber als normal, aber doch weicher als bei einfacher Stauungsmilz. Infarcte oder Blutungen oder Abscesse sind nicht zu sehen.

In beideu Nieren zahlreiche stecknadelkopforosse und darunter grosse Blutungen, welche grossentheils in der äussersten Corticalzone liegen und vielfach durcligelbe Centren ausgezeichuet sind. Andererseits finden sich da und dort auch kleinste gelb-weisse Herdchen, die nur von einem ganz schmalen hälnorrhagischen Saum umgebeu sind.

Auf der Magenschleimhaut zahlreiche kleine Blutungen mit entsprechend grossen Erosionen. Im übrigen Digestionscanal nichts nennenswerthes. Die Leber ziemlich blutreich, aber ohne Blutungen oder sonstige herdförmige Veränderungen.

Gehir’ı ziemlich hyperämisch und ödematös, aber keine Blutungeı oder sonstige Herde in seiner Substanz nachzuweisen.

Bericht über die bacteriologische Untersuchung: Sclool während der Section wurden Deckglaspräparate von den endocardialen Anflagerungen angelegt, die bei der Farbung zahlreiche streptococcen in mikroskopischer Reincultur erkemuen liessen. Desgleiclen zeigteu die mit dem gleichen Material beschickten Agar-Agarplatten, nachdem sie einen 'Tag im Brutofen bei $37^{\circ}$ gelialten waren, massenhafte Streptococcencolonieen, von denen einige entnommen und auf Gelatinebouillon übertragen wurden, woselbst sie eine absolute Reincultur von Streptococcen lieferten. Schnittpräparate wurden von den in Alkohol gehärteten, mit den Auflagerungen versehenen Theilen des Endocard sowohl, als auch von den schon makroskopisch aufgefallenen Nierenherdchen angelegt und nach dem Gram-Günther'schen Verfahren gefärbt. Die Endocardpräparate zeigten fibrinöse Auflagerungen, die von reichlichen Streptococcenmassen durchsetat waren; das darunter liegende Gewebe zeigte sich stellenweise nur wenig, an anderen Stellen dagegen recht stark kleinzellig infiltrirt. Eine Nekrose des Klappengewebes war nicht nachzuweisen. Die Nierenschnitte wiesen zahlreiche Streptococcenembolieen der kleinen Arterien vorzugsweise im Gebiete der Nierenrinde auf, an die sich theils kleinere, theils grossere Blutungen, theils ausgedehntere Epithelnekrosen der Tubuli contorti angeschlossen hatten, während die Glomeruli dieser Theile, sowie die geraden Harncanälchen durchweg deutliche Kerntinction zeigten. In der Umgebung der Coccenembolie war nur eine ganz geringe entzündliche Reaction vorhanden.

Die Krankheitsdauer betrug in diesem Falle nur 75 Stunden; die Aufnahme des Patienten in die Poliklinik geschah erst etwa 60 Stunden nach dem Anfang. Man vermuthete eine noch nicht wandständig und dadurch sicher nachweisbar gewordene Pneumonie.
Anhaltspunkte für diese Diagnose bot der ganz plötzliche Anfang mit Schüttelfrost und hohem Fieber, sowie das Verhältniss zwischen Athmung und Puls 1:2,8.

Die beiden mitgetheilten Beobachtungen sind geeignet, die Bedeutsamkeit älterer Erkrankungen des Herzens darzuthun.

Im ersten Falle war schon seit vielen Jahren ein Herzleiden vorhanden, welches sich schleichend entwickelt zu haben scheint. Man könute nach den Angaben der Kranken geneigt sein, als Ursache einen acuten Gelenkrheumatismus anzunehmen, aber ebenso gute, vielleicht bessere Gründe sprechen dafür, dass es sich von Anfang an um septische Infection gehandelt hat. Ist schon die Aussage der Patientin, sie habe an Gelenkschmerzen gelitten, so wenig bestimmt, dass daraus nicht viel zu entnehmen ist - ebenso gut können es Schmerzen in den Gliedern gewesen sein - so ist besonders gegen das vorwiegende Ergriffensein der Gelenke eine Angabe geltend zu machen: die Schmerzen sollen bei dem Gehen geringer gewesen sein als bei dem Sitzen. Nur zeitweilig wurden sie so hochgradig, dass das Bett aufgesucht werden inusste.

Bei dem acuten Gelenkrheumatismus beschränkt sich die Erkrankung auf das Gelenk selbst und seine nächste Umgebung, so dass jede Bewegung desselben äusserst schmerzhaft ist; eine $\mathrm{Ab}$ schwächung des Schmerzes beim Gehen dürfte wohl kaum vorkommen. Langsamer verlaufende, in den Knochen mehr als in den Gelenken localisirte, schwächere septische Infection dagegen kann gar wohl dieses Verhalten zeigen. Während der letzten Erkrankung war nun ganz unzweideutig neben einer hin und wieder auftretenden Gelenkschwellung die Betheiligung der Knochen nachweisbar, früher wurde nicht darauf untersucht.

Bei der Obduction fanden sich vielfach Verwachsungen der beiden Pleuren unter einander, derselben mit dem Herzbeutel, sowie der Leberoberfläche mit dem Zwerchfell; alles stammte aus älterer Zeit. Das ist ein Befund, wie er bei der langsam verlaufenden Sepsis häufig angetroffen wird. Sind doch die umschriebenen Reibegeräusche über den von der Brusthöhle umschlossenen Organen, sowie ïber Leber und Milz diagnostisch von hoher Bedeutung. Zudem fand sich noch eine Pleuraerkrankung neuesten Datums: eine frische, sammtartige fibrinoide Quellung des Pleuragewebes längs des hinteren stumpfen Lungenrandes und von da bis zum Hilus sich erstreckend an der rechten Seite. Seit dem Sommer 1878 hatte die Kranke Herzklopfen; im December wurde die vollausgebildete Insufficienz der Mitralis und die Stenose des Ostium venosum sinistrum mit der consecutiven Hypertrophie des rechten Herzens nachgewiesen.

Bei dem heutigen Stande der Lehre von der Endocarditis dürfte es erlaubt sein, mindestens von diesem Zeitpunkt an die Infection mit Eitercocen bei unserer Kranken zu datirell.

Die folgenden Lebensjahre waren ausser den durch die bleibenden Veränderungen am Herzen gesetzten dauernden Schädigungen der Arbeitsfähigkeit noch von wiederholten Anfällen acuten Erkrankens heingesucht. Aus den nicht eben genauen Angaben dürfte zu entnehmen sein, dass es sich im wesentlichen um das alte Uebel gehandelt hat. Das letzte mal führte dasselbe dann zum Tode, aber erst nachdem ein nahezu halbjähriges Krankenlager vorausgegangen war.

Ganz anders im zweiten Falle:

Von fiüheren Erkrankungen ist nichts bekannt, es lässt sich nicht bestimmen, wann die Mitralis und ihr Ostium ergriffen worden sind. Eine genügende Compensation war durch die geringere Hypertrophie des rechten Ventrikels gegeben; der mit starker Muskulatur ausgerüstete Mann blieb durchaus arbeitsfähig. Ein stürmischer Verlauf der neuen Erkrankung führte in weniger als 3 Tagen das Ende herbei. Die Leichenöffiung zeigte neben den alten Veränderungen am Herzen eine ganz frische Endocarditis und von ihr ausgegangene Mikrobenembolie. Auch die hämorrhagischen Herde in der rechten Lunge enthielten, wie nachträglich bemerkt sein soll, massembaft Streptococcen.

Die Verschiedenheiten im Verlauf der Erkrankungen dürften kaum darauf zu beziehen sein, dass im ersten Falle Staphylococcen, im zweiten Streptococcen vorhanden waren. Hat doch die Erfallrung gelehrt, dass diese wie jene die allerbösartigsten Infectionen hervorrufen können. Wohl aber ist die Eigenthümlichkeit, welche die Staphylococcen bei der Cultur zeigten, hervorzuheben: Das geringe Peptonisirungsvermögen, welches sich durch die äusserst langsam vor sich gebende Verflüssigung der Nährgelatine kund gab. Von anatomischer Seite wird damit die geringfügige entzündliche Reaction der ergriffenen Gewebstheile in Beziehung gesetzt, und es dürfte auch wohl kein Grund dagegen sprechen, dass man den so ungewöhnlich langen Verlauf der Gesammterkrankung mit der nicht hohen Virulenz der inficirenden 
Coccen in Verbindung bringt. Anders bei der Streptococceninfection. Hier dürfte der so äusserst rasche Verlauf genügen, uın die lochgradige Giftigkeit der inficirenden Mikroben ausser allen Zweifel $\mathrm{zu}$ stellen. Dass nur an den Herzklappen schwerere entzündliche Veränderungen $\mathrm{zu}$ finden waren, in den metastatischen Herden dagegen nicht, erklärt sich ungezwungen aus der kulzen Dauer des Verlaufs.

Nicht nur entzündlicbe Vorgänge, die zur Gewebszerstörung von sich aus führten und auf Ansiedelung der Mikroben an bestimmten Orten beruhen, haben in unseren Fällen das Krankheitsbild beherrscht. Neben ihnen kam sicher die Intoxication, welche durch die unter dem Eiufluss der Mikroben gebildeten Toxine erzeugt wird, zur Geltung. Es muss einer späteren Zeit die Entscheidung darüber vorbehalten bleiben, was auf unmittelbare Wirkung der das Zellenleben vernichtenden Spaltpilze, was auf die dabei entstehenden chemischen Erzeugnisse von den, den Zusammenhalt des Menschenkörpers bedrohenden Schädigungen zurïckzuführen ist. Wahrscheinlich bleibt es, soweit wir bis jetzt einen Ueberblick gewinnen können, dass die Allgemeinstörungen und besonders auch das Fieber den Toxinen zukommen.

Dass eine Endocarditis sich zu wiederholen vermag, ist eine längst festgestellte Thatsache - hat man doch den Ausdruck "recurrirende Endocarditis" schon seit längerer Zeit in die Pathologie eingeführt. Offen bleibt aber die Frage, ob es sich dabei um eine erneute Aufnahme der inficirenden Mikroben von aussen her, welche an dem schon früher erkrankten Herde nur einen günstigen Brutplatz finden, handelt, oder aber, ob an Ort und Stelle Keime zurückgeblieben sind, welche unter günstigen Bedingungen sich wiederum zu entwickeln vermögen. Das letztere wird für die Tuberkelbacillen kaum zu bestreiten sein. Hier gebell die bacteriologischen Untersuchungen eine sichere Grundlage für das Urtheil ab. Eitercoccen sind in solchen alten Herden, die zu ruhen schienen, bisher nicht nachgewiesen. Es ist also eine bestimmte Entscheidung gegenwärtig unmöglich. Vom klinischen Standpunkte aus wird man eher geneigt sein, mit dem Verweilen der Krankheitserreger an der einmal ergriffenen Stelle zu rechnen. Prof. Jürgensen (cf. Lehrbuch 2. Auflage p. 408 ff.) wenigstens neigt bei ausgedehnterer Erfahrung mehr und mehr sich dieser Auffassung zu und möchte die Analogie mit der tuberculösen Infection immer stärker betonen, welche er bereits früher hervorgehoben hat (l. c. p. 290). 